\title{
« La manière la plus efficace de maintenir la tranquillité »?
}

La place de la gendarmerie impériale dans le dispositif français du nord de l'Espagne (1810-1814)

\section{Gildas Lepetit}

\section{(2) OpenEdition}

Journals

Édition électronique

URL : https://journals.openedition.org/ahrf/9103

DOI : 10.4000/ahrf.9103

ISSN : 1952-403X

Éditeur :

Armand Colin, Société des études robespierristes

Édition imprimée

Date de publication : 1 juin 2007

Pagination : 87-100

ISSN : 0003-4436

Référence électronique

Gildas Lepetit, « « La manière la plus efficace de maintenir la tranquillité »? », Annales historiques de la Révolution française [En ligne], 348 | Avril-Juin 2007, mis en ligne le 01 juin 2010, consulté le 03 mai 2022. URL : http://journals.openedition.org/ahrf/9103 ; DOI : https://doi.org/10.4000/ahrf.9103

Ce document a été généré automatiquement le 3 mai 2022.

Tous droits réservés 


\section{« La manière la plus efficace de maintenir la tranquillité »?}

La place de la gendarmerie impériale dans le dispositif français du nord de l'Espagne (1810-1814)

\section{Gildas Lepetit}

1 Le 14 juillet 1913, Raymond Poincaré remet un drapeau à la gendarmerie départementale. Le 4 novembre suivant, il est décidé qu'il serait orné du nom de quatre batailles, parmi lesquelles Villodrigo, au cours de laquelle la 1ère légion de gendarmerie d'Espagne enfonce la ligne des dragons-rouges anglais le 23 octobre 1812. Magnifié par une institution en quête constante de légitimité militaire, le souvenir de ce combat exhume, après un siècle d'oubli, la participation de la gendarmerie à la campagne d'Espagne.

2 En effet, le 24 novembre 1809, un décret impérial ordonne la création d'un corps de vingt escadrons de gendarmerie destinés à servir dans les provinces du nord de l'Espagne. Plus de deux ans après le franchissement des Pyrénées par le corps expéditionnaire de Junot $^{1}$, Napoléon décide donc d'envoyer la gendarmerie pour pacifier cinq régions: la province de Santander, les provinces basques, la Navarre, l'Aragon et la Castille. Après des difficultés initiales - tant humaines que matérielles -, les escadrons pénètrent dans la péninsule dès le mois de mars $1810^{2}$. Cette précipitation est le signe d'une urgence : Napoléon souhaite utiliser l'arme et lui faire jouer un rôle prépondérant. Cette perspective, peu étudiée par l'historiographie, met en lumière l'emploi de la gendarmerie dans le cadre particulier de sa mission combattante.

Mémoire et histoire

3 Le XIX ${ }^{\mathrm{e}}$ siècle est celui des mémorialistes. Comme l'écrit Jean Tulard, « nulle époque n'a suscité la publication d'un nombre aussi élevé de mémoires que le Consulat et l'Empire $»^{3}$. Au milieu de cette littérature prolifique, la gendarmerie fait exception. Arme de l'écrit par excellence ${ }^{4}$, elle oppose un mutisme paradoxal quand il s'agit de raconter son activité. Peu de ses membres ont évoqué leur carrière en l'offrant à la lecture du public. Or, les gendarmes participent à l'ensemble des campagnes napoléoniennes. Tous les pays du vieux continent les voient circuler ou stationner. 
Qu'ils soient installés au sein des brigades créées dans les pays nouvellement annexés ou chargés d'assurer la discipline des armées en campagne, ils montrent leur uniforme aux quatre coins de l'Europe, de Madrid à Moscou, de Hambourg à Rome.

4 Cette carence n'est pas comblée par les autres témoins de son action. En effet, les militaires ayant servi à leurs côtés ne les mentionnent que très rarement et, le plus souvent, en termes fort peu élogieux. Le capitaine Parquin, par exemple, évoque une rixe en sortie de cabaret avec deux gendarmes « échauffés par le vin » de la garnison de Valladolid, au cours de laquelle le chef d'escadron de Vérigny est tué5. Dans le même ordre d'idée, le baron Ernouf rapporte qu'à son retour en France, il prend place dans un convoi escorté pour partie par «un régiment de gendarmes à pied de formation nouvelle, beaux jeunes hommes bien équipés, mais bien mal disciplinés ", dont "au moins un bon tiers [...] étaient ivres à ne pas se tenir $»^{6}$. Le capitaine Desboeufs, quant à lui, se montre certes un peu plus disert sur les membres de l'institution, mais toujours aussi sévère. Il retranscrit notamment les propos du général Pâris qui reproche aux gendarmes de s'être rendus «lâchement » à Huesca. Plus grave encore, il les accuse de trahison, après le retour des légions sur le territoire français, soulignant que les gendarmes auraient facilité le commerce des denrées alimentaires en direction de «l'ennemi $»^{8}$.

5 Ces assertions, difficilement vérifiables', présentent les gendarmes d'Espagne sous l'aspect de soudards chez lesquels la couardise le dispute à l'alcoolisme! En d'autres termes, les mémorialistes se sont le plus souvent contentés de décrire les agissements des gendarmes plutôt que leurs éventuelles actions.

D'autres acteurs de la campagne d'Espagne se montrent toutefois plus indulgents avec l'institution. Le général Marbot, par exemple, salue le service de l'arme dans la péninsule ${ }^{10}$. Cependant, il n'opère aucune distinction entre les différentes unités de gendarmerie, les considérant comme faisant toutes partie de la 1ère légion de gendarmerie à cheval qui semble bénéficier d'une estime raisonnable au sein des troupes de ligne ${ }^{11}$.

7 En réalité, l'institution pâtit de l'absence d'écrits émanant des généraux sous les ordres desquels elle eut à servir. Ni Thouvenot, ni Reille, ni, à plus forte raison, d'Agoult ou Dufour n'ont raconté leur campagne d'Espagne. Seul le maréchal Suchet a produit des Mémoires. Les témoignages qui nous sont parvenus sont donc, pour leur grande majorité, le fruit de personnes n'ayant pas une vision globale du service de la gendarmerie.

8 Paradoxalement, il faut se tourner vers les Espagnols pour trouver des mentions "positives » sur les pandores. Les envolées de Espoz y Mina contre le chef d'escadron Mendiry sont là pour en attester ${ }^{12}$. Quoi qu'il en soit, cette absence relative des gendarmes dans les mémoires de l'époque les pousse inexorablement vers l'oubli.

9 Certes, la campagne d'Espagne est un des thèmes d'étude privilégiés au cours du XIX ${ }^{e}$ siècle. Le foisonnement des témoignages et le retentissement du soulèvement espagnol en sont en grande partie les raisons. Pourtant, sur l'ensemble de ces travaux, la gendarmerie est généralement absente. Ni Grandmaison, ni Sarazin, ni Grasset ne la mentionnent. Ils s'intéressent plus à la guerre de masse, aux charges épiques et meurtrières, qu'à un corps dont les compétences combattantes sont très récentes voire limitées - et dont la faiblesse numérique les empêche de participer à des batailles de grande envergure. La majorité de l'historiographie de la campagne d'Espagne est monopolisée par les études sur les grandes figures militaires françaises, sur les 
intentions de Soult, sur la pacification par Suchet, quand la gendarmerie n'a à sa tête qu'un simple général. On privilégie volontiers l'histoire globale et les quatre mille gendarmes sont noyés dans une masse de régiments, de divisions et de corps d'armée au milieu desquels ils sont, numériquement, quantité négligeable.

En réalité, il faudra attendre que la gendarmerie étudie elle-même ce pan de son histoire pour remettre en lumière l'action des gendarmes en Espagne. En 1898 - soit près de quatre-vingt-dix ans après les faits -, le capitaine de gendarmerie Emmanuel Martin $^{13}$ exhume la gendarmerie d'Espagne de l'abîme dans lequel l'histoire l'avait projetée. Pendant une grande partie du XXe siècle, son ouvrage est le seul consacré au service de l'arme dans la péninsule, avant que, au début des années 1970, les travaux du sous-lieutenant Bernard Charron ne viennent donner une perspective nouvelle ${ }^{14}$.

11 On ressent d'ailleurs ce renouveau avec l'apparition de la gendarmerie d'Espagne dans les travaux de Jean Sarramon achevés en $1970^{15}$. Les auteurs anglo-saxons se sont également intéressés à la gendarmerie d'Espagne. Dans Rod of Iron, Don W. Alexander mentionne à de nombreuses reprises l'action de la gendarmerie dans le cadre de la pacification de l'Aragon ${ }^{16}$. L'avènement d'études plus locales et de monographies sur les régions concernées par la contre-guérilla provoque un renouvellement de l'historiographie de la gendarmerie d'Espagne ${ }^{17}$.

La gendarmerie d'Espagne, une quantité négligeable?

12 Loin de l'image traditionnelle de la guerre de masse napoléonienne, l'arme sert dans la péninsule par petits détachements à effectifs restreints. Au terme du décret de novembre 1809, chaque unité de la gendarmerie d'Espagne dispose d'une force de sept officiers et deux cents gradés et gendarmes, répartis en deux composantes, l'une à pied et l'autre à cheval ${ }^{18}$. Les fantassins sont au nombre de cent vingt et les cavaliers de quatre-vingts. La gendarmerie de l'Empire ne pouvant à elle seule assumer une telle ponction ${ }^{19}$, il est procédé à des recrutements au sein des troupes de ligne de militaires susceptibles de remplir les conditions nécessaires pour l'intégrer.

Quelques mois plus tard, le système des escadrons ayant démontré ses limites, les autorités impériales décident de les regrouper en légions. La première d'entre-elles est formée en Catalogne par le décret du 6 juillet $1810^{20}$. Suivent la légion de gendarmerie à cheval de Burgos précédemment citée, et quatre autres légions regroupant les vingt escadrons préexistants.

14 Attendue avec impatience par certains généraux gouverneurs militaires ${ }^{21}$, la gendarmerie impériale pénètre en Espagne au mois de mars 1810. Cet engouement initial n'est pas feint. Les provinces septentrionales de l'Espagne souffrent d'un manque cruel de troupes. Elles sont constamment traversées par des régiments envoyés pour renforcer les autres corps de l'armée, mais très peu y demeurent. Le maréchal Suchet relate ainsi un épisode symptomatique :

«[À la fin de l'année 1810,] environ trente mille hommes de troupes venant de France étaient entrés en Biscaye et en Navarre. Le général Reille avait le projet de profiter de leur séjour pour détruire les bandes du pays [...], mais il n'eut pas le temps d'effectuer cette résolution; ces troupes furent presque toutes envoyées en Portugal $»^{22}$.

15 Par ailleurs, l'arrivée de gendarmes permet aux autorités françaises de s'appuyer sur des militaires aguerris ${ }^{23}$. En effet, toute personne désireuse d'intégrer l'arme doit disposer d'une expérience militaire d'au moins quatre campagnes dans la ligne. Ainsi, pour les escadrons concernés, les sous-officiers et gendarmes en totalisent en moyenne 
huit ${ }^{24}$ et les officiers plus de sept ${ }^{25}$. On est bien éloigné des reproches formulés par le général Marbot sur l'extrême jeunesse des soldats envoyés par Napoléon dans la péninsule ${ }^{26}$. Par ailleurs, les gendarmes sont, non seulement des militaires expérimentés, mais également des militaires français à la fidélité éprouvée ${ }^{27}$, bien que cela n'ait pas empêché certains d'entre eux de déserter ${ }^{28}$.

Pour autant, ce renfort ne modifie pas considérablement les effectifs français dans le nord de l'Espagne. La place prise quantitativement par la gendarmerie reste marginale.

Tableau des effectifs militaires dans les provinces septentrionales de l'Espagne au 15 novembre 1810 et au 1 er décembre $1811^{29}$.

\begin{tabular}{|c|c|c|c|c|c|c|c|}
\hline & \multicolumn{2}{|l|}{15 novembre 1810} & \multicolumn{5}{|l|}{$1^{\text {er }}$ décembre 1811} \\
\hline & $\begin{array}{l}\text { Effectifs de } \\
\text { gendarmerie }\end{array}$ & $\begin{array}{l}\text { Effectifs } \\
\text { globaux }\end{array}$ & $\begin{array}{l}\text { Proportion de } \\
\text { gendarmes (en \%) }\end{array}$ & \begin{tabular}{l|l} 
e & Effect \\
genda
\end{tabular} & herie ${ }^{\text {de }}$ & $\begin{array}{l}\text { Effectifs } \\
\text { globaux }\end{array}$ & $\begin{array}{l}\text { Proportion de } \\
\text { gendarmes }\end{array}$ \\
\hline Santander & 399 & 3609 & 11,1 & 445 & 4816 & 9,2 & \\
\hline $\begin{array}{l}\text { Provinces } \\
\text { basques }\end{array}$ & 808 & 6719 & 12 & 1289 & 10697 & 12,1 & \\
\hline Navarre & 834 & 9924 & 8,4 & & - & - & \\
\hline Aragon & 1035 & 1750 & 59,1 & 1094 & 6074 & 18 & \\
\hline Total & 3076 & 22002 & 14 & 2828 & 21587 & 13 & \\
\hline
\end{tabular}

18 Ainsi, un peu moins d'un militaire sur sept présents dans le nord de la péninsule ibérique est un gendarme. Certes, cette proportion peut sembler faible, mais les gendarmes ont l'avantage, par rapport aux troupes de ligne, de demeurer dans les provinces. Ils représentent les éléments d'occupation permanente de la présence française. Les différentes réorganisations connues par la gendarmerie d'Espagne ne modifient pas réellement cet état de fait $^{30}$. Un an plus tard, le pourcentage de gendarmes n'a guère évolué.

19 Aussi, malgré une augmentation substantielle de ses effectifs, la gendarmerie demeuret-elle un élément quantitativement mineur du dispositif français. Cependant, il n'en est pas de même pour ce qui concerne spécifiquement la cavalerie ${ }^{31}$. 
Tableau des effectifs de chevaux dans les provinces septentrionales de l'Espagne au 15 novembre 1810 et au 1 er décembre 1811 .

\begin{tabular}{|c|c|c|c|c|c|c|c|c|c|}
\hline & \multicolumn{4}{|c|}{15 novembre 1810} & \multicolumn{5}{|c|}{$1^{\text {er }}$ décembre 1811} \\
\hline & $\begin{array}{l}\text { Effectifs de } \\
\text { gendarmerie }\end{array}$ & $\begin{array}{l}\text { Effectifs } \\
\text { globaux }\end{array}$ & $\begin{array}{l}\text { Proportion } \\
\text { chevaux } \\
\text { gendarmerie } \\
\text { (en \%) }\end{array}$ & $\begin{array}{l}\text { de } \\
\text { de }\end{array}$ & $\begin{array}{l}\text { Effectifs } \\
\text { gendarmer }\end{array}$ & rie & $\begin{array}{l}\text { Effectifs } \\
\text { globaux }\end{array}$ & $\begin{array}{l}\text { Pro } \\
\text { che } \\
\text { ger } \\
\text { (en }\end{array}$ & $\begin{array}{ll}\text { tion } & \text { de } \\
\text { cmerie } & \text { de } \\
& \end{array}$ \\
\hline Santander & 132 & & 151 & 87,4 & & 75 & 13 & & 57,7 \\
\hline $\begin{array}{l}\text { Provinces } \\
\text { basques }\end{array}$ & 321 & & 1063 & 30,2 & & 332 & 42 & & 78,1 \\
\hline Navarre & 284 & & 950 & 29,9 & - & & - & & - \\
\hline Aragon & 413 & & 413 & 100 & & 213 & 72 & & 29,3 \\
\hline Total & 1150 & & || 2577 & 44,6 & & 620 & 12 & & 48,3 \\
\hline
\end{tabular}

Les escadrons de gendarmerie représentent donc près de la moitié de la cavalerie française dans ces régions. En Aragon, en novembre 1810, ils sont même les seules forces à cheval disponibles. Cette importance de la composante à cheval de la gendarmerie ne cesse d'augmenter, comme l'atteste l'état de la remonte un an plus tard.

21 Bien que les effets du décret de formation de la légion de Burgos se soient faits sentir dans les effectifs des escadrons en cavalerie, l'importance de la gendarmerie ne faiblit pas dans ce domaine.

De la spécificité gendarmique en Espagne

Outre le point de vue quantitatif, l'envoi de la gendarmerie en Espagne présente un double intérêt. Il permet, dans un premier temps, d'employer un personnel adapté aux missions de rétablissement de l'ordre et de surveillance de route. Par ailleurs, il offre la possibilité de pourvoir au remplacement des troupes de ligne présentes dans les garnisons pour les réaffecter à des missions plus combattantes comme l'envoi sur des fronts actifs ou la participation à des colonnes mobiles destinées à pourchasser les bandes armées espagnoles.

Cependant, si la présence de la gendarmerie est nécessaire pour ramener le calme dans les provinces septentrionales de la péninsule, elle n'est en aucun cas suffisante. Les généraux français en ont conscience ${ }^{32}$. D'ailleurs, l'expérience tentée en Aragon après le départ du III ${ }^{e}$ Corps de l'armée d'Espagne pour la province de Valence donne des résultats catastrophiques ${ }^{33}$ malgré la confiance - toute relative pourtant - de Suchet en la gendarmerie ${ }^{34}$.

24 Le manque endémique de troupes oblige cependant les généraux français à employer les gendarmes comme de simples combattants, faisant pour la plupart fi de leur spécificité. Dès lors, les autres prérogatives traditionnellement dévolues à l'arme en souffrent. Les gendarmes peuvent sans difficulté remplir des missions combattantes et 
de rétablissement de l'ordre. Ils ont quasiment tous participé à plusieurs campagnes dans les armées révolutionnaires et impériales. Ainsi, ils escortent des convois de fonds ou d'armement, entrent dans la composition des colonnes mobiles qui pourchassent les insurgés ou prélèvent les contributions tant en nature qu'en argent, collectent et analysent le renseignement, assurent la police dans les armées et tiennent des garnisons $\mathrm{s}^{35}$.

Si l'on excepte les missions de maintien de l'ordre aux armées, la gendarmerie sert en grande partie selon les mêmes principes que les troupes de ligne. En revanche, il existe des domaines où elle est employée plus spécifiquement.

De nombreux généraux ont ainsi essayé d'intégrer des Espagnols au sein de leur dispositif de pacification. En Aragon, par exemple, Suchet crée des compagnies de fusiliers ou de gendarmes aragonais ${ }^{36}$. Mais ces dernières ne sont pas assimilées aux autres troupes françaises. L'encadrement y est espagnol, au même titre que la totalité des effectifs. Dans le gouvernement de Biscaye, le général Thouvenot agit de manière différente. Plutôt que de former des unités exclusivement espagnoles, il recrute des autochtones au sein des escadrons de gendarmerie. C'est ainsi que quatre d'entre eux sont dotés de deux brigades de "gendarmes cantabres", soit vingt-cinq hommes chacun ${ }^{37}$.

L'objectif de ces recrutements est double. Dans un premier temps, il permet aux gendarmes de disposer de jeunes gens qui maîtrisent, non seulement la langue, mais également la géographie du pays. Par ailleurs, outre cette mission de guidesinterprètes, les Cantabres sont une vitrine pour la France. Ils doivent faire de la propagande auprès des populations et participer à l'amélioration de l'image des Français. Au sein de ces brigades sont recrutés des jeunes gens des provinces basques, mais aussi d'anciens insurgés. Ainsi, Barroutia est engagé dans cette unité, car le duc d'Istrie espère que «le bon accueil qu'il [lui] ferait entraînerait la soumission d'autres chefs $»^{38}$. De même, pour ce qui concerne la mission de renseignement, étant basques, ils disposent de réseaux de relations et donc, indirectement, d'informations qu'ils peuvent mettre à la connaissance de la gendarmerie d'Espagne.

Cette expérience d'acculturation n'est pas un véritable succès. De nombreux Cantabres désertent avec armes et bagages ${ }^{39}$. Bien que cela ne soit pas avéré, il n'est pas impossible qu'ils en profitent pour rejoindre les forces insurgées. Certains d'entre eux servent malgré tout avec zèle. Ainsi, six ont trouvé la mort au service de l'empereur. Il convient de nuancer ce chiffre, puisque trois sont décédés à l'hôpital dans des circonstances inconnues ${ }^{40}$. Ce nombre, mis en perspective avec celui des déserteurs, donne un aperçu de l'attachement des Cantabres aux aigles impériales. Sept fois plus quittent le service de l'empereur par désertion que par décès! Ils sont cependant encore vingt-sept présents au sein des quatre premiers escadrons de la gendarmerie d'Espagne au 1er juin $1813^{41}$. Le 8 janvier suivant, ils ne sont plus que seize à s'être exilés en France ${ }^{42}$. L'un d'entre eux, le sous-lieutenant Barroutia, pourtant ancien membre d'une bande de guérilleros, continue d'être considéré comme un dangereux bonapartiste en $1817^{43}$ !

Sur le plan du prosélytisme, l'échec est encore plus cuisant. La continuelle augmentation des effectifs des insurgés et l'aide croissante que la population leur apporte soulignent la permanence de l'hostilité des Espagnols à l'égard des Français. Ce désamour se traduit également par la piètre qualité du renseignement recueilli par les gendarmes, signe patent du peu d'intégration de l'institution ${ }^{44}$. 
30 On aurait pu imaginer que la gendarmerie soit également la seule à exercer la justice prévôtale, rétablie exceptionnellement à l'occasion de la campagne d'Espagne. Supprimée en 1790, cette dernière permettait aux membres de l'ancienne maréchaussée de juger sur place un individu pris dans le cas de la flagrance. Dans les provinces basques, par exemple, le général Thouvenot ordonne dès mars 1810, date de l'arrivée des escadrons, que les Espagnols sans uniforme pris les armes à la main seront exécutés ${ }^{45}$. En Navarre, le général Reille attache des gendarmes « à toutes les colonnes et [ordonne] aux chefs de cette arme de juger prévôtalement tout individu reconnu comme ayant favorisé les brigands $»^{46}$. Avec ce rétablissement, déjà réalisé lors de la pacification de l'ouest de la France en $1794^{47}$, se pose invariablement la question du statut juridique des guérilleros. En l'absence d'un statut de soldats clairement établi, l'application de la justice prévôtale peut éventuellement se justifier juridiquement, mais comment agir dès lors que les bandes se militarisent, prennent des noms de bataillons ou de régiments et revêtent des uniformes? En janvier 1812, le général Thouvenot fait part de son désarroi à ce sujet au maréchal Berthier. Il lui expose que « les bandes étant maintenant réunies et formant une espèce d'organisation militaire, je prie Votre Altesse Sérénissime de vouloir bien me faire connaitre si les hommes qui pourraient tomber dorénavant entre nos mains doivent être traités comme brigands et jugés comme tels, ou considérés comme prisonniers de guerre, ne pouvant moi-même décider une telle question, qui peut donner lieu à des actes de réciprocité, que je crois devoir soumettre à Votre Altesse $»^{48}$. Malgré cela, les exécutions prévôtales perdurent ${ }^{49}$. L'exercice de cette justice expéditive est-elle l'apanage de l'institution? Cette question ne peut être tranchée. Les imprécisions des sources ne donnent qu'exceptionnellement la composition des pelotons chargés de l'exécution des insurgés. On peut cependant penser que, sous couvert de cette «justice», des militaires non-gendarmes se soient laissés aller à fusiller des guérilleros pris les armes à la main.

Un bilan contrasté

31 Si l'on ne peut réellement mettre en exergue une réelle originalité gendarmique en terme de missions, les résultats obtenus ne sont pas négligeables. Certes la gendarmerie ne fait pas « des merveilles » comme le pense le général Thouvenot ${ }^{50}$, mais, à l'inverse, son service n'a pas été «insignifiant » comme le prétend Suchet ${ }^{51}$. En réalité, son efficacité est intimement liée aux circonstances. À son arrivée, elle ne doit lutter que contre "quelques petites bandes " ${ }^{52}$. Elle le fait avec succès : elle en disperse beaucoup et arrête de nombreux chefs guérilleros. Ainsi, dès le 17 mars 1810, le chef de bande Cuco est tué à Elorio par un détachement de gendarmes ${ }^{53}$. Au début du mois d'avril, le plus célèbre guérillero de la Navarre, Javier Mina, est arrêté ${ }^{54}$. Ortiz et Ugarte sont faits prisonniers entre février et juin $1811^{55}$. Ces bons résultats, s'ils ne masquent pas complètement les carences d'une gendarmerie en partie sclérosée par l'âge excessif de son personnel, pâtissent par la suite du recul de l'influence française dans la péninsule. En peu de temps, les troupes impériales perdent l'initiative de la guerre. Comme le résume avec beaucoup de justesse le général Buquet en mai 1813: "la guerre a tellement changé en un an que les insurgés ont pris notre place et nous la leur $»^{56}$. Pour preuve, les effectifs des insurgés approchent, selon des estimations françaises, les vingt mille hommes en mai $1813^{57}$. En 1810 et 1811 , les troupes impériales se battent pour pacifier le territoire conquis; en 1812 et 1813, il ne s'agit plus de pacification mais de préservation. L'accroissement du nombre de guérilleros oblige les autorités françaises à regrouper leurs forces, abandonnant du même coup les campagnes - et donc les principales ressources alimentaires - aux insurgés. Ce déclin de la suprématie impériale 
est également patent dans la multiplication des tentatives de coups de main contre des garnisons françaises. La ville de Bilbao, par exemple, subit cinq tentatives d'invasion pour la seule année $1813^{58}$ dont plusieurs aboutissent à la réoccupation temporaire de la ville par les Espagnols.

D'ailleurs, sur la fin de la période, les gendarmes sont de plus en plus employés comme les troupes de ligne. $\mathrm{Si}$, dans les premiers temps, les combats en rase campagne impliquant des gendarmes sont rares, ils se multiplient au fur et à mesure de la militarisation des bandes. Les sièges de garnisons ne sont rien d'autres que des actes de guerre. Nous avons précédemment mentionné les attaques subies par la ville de Bilbao en 1813. À chaque fois que la ville est occupée par les Espagnols, les gendarmes participent à la reconquête. De même, la bataille de Villodrigo, inscrite sur le drapeau de l'institution et seul événement où la gendarmerie est donnée dans la ligne, se déroule en octobre 1812 soit quelques mois à peine avant le retrait des troupes françaises au nord des Pyrénées. En cas de nécessité, les autorités ont su se souvenir du statut militaire de l'arme et s'en servir pour combler l'insuffisance des troupes de ligne.

Si les missions confiées à l'arme ne marquent pas une forte spécificité gendarmique, la fréquence de son emploi laisse entrevoir toute son importance au sein du dispositif français. Une étude du bilan humain de cette campagne pour les escadrons de gendarmerie d'Espagne rend compte du prix payé par l'institution. Sur les 6500 hommes qui se sont succédé dans la péninsule, plus de huit cents ont trouvé la mort dans le nord de l'Espagne ${ }^{59}$. Bien qu'important, ce chiffre est, selon toute vraisemblance, encore très inférieur à la réalité. Outre les lacunes archivistiques, il nous est impossible de connaitre le nombre exact de gendarmes morts dans leurs foyers suite aux séquelles de leur séjour en Espagne, ni celui des hommes qui ne sont pas rentrés des pontons anglais. Rappelons à ce propos que plus de mille deux cents gendarmes ont été faits prisonniers au cours de cette campagne. Quoi qu'il en soit, plus de $12 \%$ des hommes ayant servi dans les escadrons y ont trouvé la mort ${ }^{60}$. Cette proportion est bien supérieure aux pertes éprouvées par la ligne. Il semble dès lors que l'arme ait payé un tribut plus lourd, en proportion, que les autres corps de troupes dans cette lutte contre l'insurrection, alors qu'elle n'a servi que deux ans et demi après le début du conflit. Si Ronald Fraser avance un bilan de quatre-vingt-dix mille hommes tués par les guérillas qui nous paraît conforme à la réalité ${ }^{11}$, Rory Muyr, quant à lui, estime à trois cent mille le nombre total de Français tués dans la péninsule ${ }^{62}$. En mettant en perspective ces deux chiffres, on se rend compte que près du tiers des pertes françaises globales ont été causées par les partisans espagnols, ce qui met davantage en relief l'importance de la lutte contre la guérilla, et donc le service de la gendarmerie.

Certes l'efficacité ne se calcule pas en nombre de morts - au contraire -, mais cette surmortalité est le signe évident d'un emploi important des gendarmes, donc d'une véritable confiance des gouverneurs, ou du manque d'effectifs rendant le recours à la gendarmerie indispensable.

D'ailleurs, la gendarmerie a-t-elle pour vocation de combattre en Espagne? Dans l'esprit napoléonien, les gendarmes doivent rétablir l'ordre et non livrer bataille. L'empereur n'a probablement pas une vision très précise de la guérilla espagnole. Il pense qu'il parviendra à la détruire en utilisant les mêmes méthodes qu'en Italie ou que dans les départements de l'ouest de la République. Dès lors, l'envoi de la gendarmerie s'impose de lui-même. Car le gendarme n'est pas un soldat comme les autres pour 
Napoléon. N'écrit-il pas à Berthier en 1812 que "deux à trois cents hommes de cavalerie de plus ou de moins ne sont rien. Deux cents gendarmes de plus assurent la tranquillité de l'armée et le bon ordre ${ }^{63}$ ? Plus largement, le gendarme est la sentinelle de la loi, son représentant en tout lieu et en tout temps. Il en est le garant. Napoléon envoie donc quatre mille "soldats de la loi » en Espagne, alors qu'il aurait pu tout autant n'y expédier que de simples militaires. Cette nuance n'a probablement pas été perçue par les gouverneurs militaires ${ }^{64}$.

Quoi qu'il en soit, il convient de ne pas garder de la gendarmerie l'image d'un corps insignifiant, se contentant de limiter l'usage des boissons alcoolisées dans les bivouacs. En Espagne, le gendarme a pris une part active, mais difficilement quantifiable, dans la lutte contre l'insurrection. Si les résultats initiaux sont satisfaisants, ils se délitent à mesure que l'influence française se fait plus indigente.

En juin 1813, la majorité des gendarmes rentre sur le territoire national, laissant près d'un millier des leurs prisonniers dans différentes garnisons. Ce retour ne signifie pas pour autant la fin des dangers ou celle des combats. S'ouvrent devant eux des missions non moins périlleuses: la lutte contre l'insoumission et la guerre contre les envahisseurs.

\section{NOTES}

1.Junot pénètre en Espagne le 17 octobre 1807.

2.À propos des difficultés rencontrées dans la réunion des effectifs des escadrons de la gendarmerie de l'armée d'Espagne, voir Gildas LEPETIT, La Gendarmerie impériale en Espagne (1810-1813) : un instrument de pacification de la Péninsule ibérique, DEA, sous la dir. de Jean-Noël Luc, Paris IV, 2002,p. 153-204.

3.Jean TULARD, Bibliographie critique des mémoires sur le Consulat et l'Empire écrits ou traduits en français, Genève-Paris, Droz, 1971, p. VII.

4.L'obligation de savoir lire et écrire est inscrite dans les textes réglementaires régissant l'arme.

5.Capitaine PARQUIN, Souvenirs de guerre (1803-1814), Paris, Le livre chez vous, 2001, p. $117-118$

6.Baron ERNOUF, Souvenirs d'un officier polonais en Espagne et en Russie, Paris, Charpentier, 1877, p. 169.

7.Marc Desboeufs, Le capitaine Desboeufs. Les étapes d'un soldat de l'Empire, Paris, Picard, 1901, p. 171.

8.Id., p. 199.

9.À propos de l'événement évoqué par Parquin, il convient de préciser que nous n'avons trouvé aucune mention de l'existence d'un chef d'escadron de Vérigny. L'alcoolisme des gendarmes est une réalité, même s'il n'est pas présent dans les proportions données par Ernouf. Voir Gildas LEPETIT, La Gendarmerie impériale dans le Vascongadas (1810-1813), l'échec dans la lutte contre la guérilla d'un corps hybride, maitrise d'histoire, sous la dir. de Jean-Noël Luc, Université de Paris IV-Sorbonne, 2001, p. 60-63. 
Enfin, si la « trahison » des gendarmes ne peut être ni avérée ni démentie formellement, le jugement porté par Pâris sur le comportement des gendarmes à Huesca semble pour le moins sévère au vu des rapports officiels relatant cette affaire. En effet, si une garnison de gendarmerie appartenant au $13 \mathrm{e}$ escadron a bien été prise par le chef de guérilleros Espoz y Mina à la fin du mois de janvier 1812, elle ne le fut qu'après cinq jours et cinq nuits de résistance. Lettre du 28 janvier 1812 du général Buquet au maréchal Berthier, SHD-DAT, C8 89.

10.Marcellin MARBOT, Mémoires du général baron Marbot, t. 2, Madrid, Essling, Torrès-Védras, Paris, Plon, 1892, 495 p.

11.La 1ère légion de gendarmerie d'Espagne dite " légion de gendarmerie à cheval », stationnée à Burgos, est créée par un décret impérial en date du 13 novembre 1810 et formée par prélèvement de gendarmes à cheval au sein des vingt escadrons initialement créés par le décret du 24 novembre 1809.

12.Francisco ESPOZ y MiNA, Memorias del general don Francisco Espoz y Mina, Madrid, 1851-52,5 t., 3 vol.

13.Emmanuel MARTIN (capitaine), La gendarmerie française en Espagne et au Portugal, Paris, Léautey, 1898, $481 \mathrm{p}$.

14.Bernard CHARRON, La participation de la gendarmerie impériale à la guerre d'Espagne, doctorat de IIIe cycle, Bordeaux III, 1972, 340 p.

15.Jean SARRAMON, La guerre d'indépendance de la péninsule ibérique contre Napoléon Ier, 1970, $14 \mathrm{vol}$.

16.Don W. AlEXANDER, Rod of Iron: French Counterinsurgency Policy in Aragon during the Peninsular War, Wilmington, Scholarly resources, 1985, $260 \mathrm{p}$.

17.Jean-Marc Lafon a soutenu en 2004 une thèse sur l'occupation française en Andalousie dans laquelle il opère une comparaison entre les méthodes de contreguérilla employées dans cette région et dans les provinces septentrionales de la péninsule. Il mentionne à cette occasion l'action de la gendarmerie. Jean-Marc LAFON, Le paradoxe andalou (1808-1812). Contre-insurrection, collaboration et résistances dans le midi de l'Espagne, doctorat d'histoire, sous la dir. Jules Maurin, Université Montpellier III, 2004. De même, Jean-Joël Brégeon y consacre quelques lignes dans son chapitre dédié à l'étude de la contre-guérilla. Jean-Joël BRÉGEON, Napoléon et la guerre d'Espagne 1808-1814, Paris, Perrin, 2006, p. 196.

18.Décret du 24 novembre 1809 formant vingt escadrons de gendarmerie en Espagne, article III, SHD-DAT, Xf 172.

19.Au 1er novembre 1809, la gendarmerie dispose en effet d'un effectif global inférieur à seize mille hommes. On comprend alors aisément les réticences napoléoniennes pour en extraire près du quart et les projeter dans la péninsule. Rapport du 12 novembre 1809 du ministre de la Guerre à l'empereur. SHD-DAT, C8 33.

20.Cette dernière ayant une histoire particulière et étant détachée des autres légions en matière de gestion, nous avons pris le parti de l'exclure de notre champ d'investigation.

21. «La gendarmerie que vous m'annoncez ne peut arriver plus à propos car sans elle j'allais me trouver sans troupe dans la province de Guipuzcoa, même pour la place de Saint Sébastien ». Lettre du 22 février 1810 du général Thouvenot au général Buquet. SHD-DAT, C8 185.

22. Maréchal SucHET, Les Mémoires du maréchal Suchet, duc d'Albuféra, sur ses campagnes en Espagne depuis 1808 jusqu'en 1814, Paris, Anselin, 1834, t. 1, p. 204. 
23.Pour plus de détails sur les caractéristiques des gendarmes envoyés en Espagne, voir Gildas LEPETIT, «Les hommes de la Gendarmerie d'Espagne », dans Force publique, actes du colloque La gendarmerie, les gendarmes et la guerre, Paris, octobre 2005, 2006, p. 31-44. 24.Plus exactement 7,9 campagnes sur un total de 468 gendarmes. 25.Plus exactement 7,3 campagnes sur un total de 67 hommes.

26." Quel spectacle pour les populations espagnoles qui accouraient de toutes parts pour contempler les vainqueurs de Marengo, d'Austerlitz et de Friedland, et ne voyaient que de chétifs conscrits pouvant à peine porter leurs sacs et leurs armes, et dont la réunion ressemblait plutôt à l'évacuation d'un hôpital qu'à une armée marchant à la conquête d'un Royaume !... ». Marcellin MARBOT, op. cit., p. 3.

27.Le général Marbot présente l'emploi de troupes étrangères comme l'une des causes des revers français dans la péninsule. Marcellin МАRBOT, op. cit., p. 482-483.

28.Si l'on excepte le 14e escadron dont les chiffres ne nous sont pas parvenus, la gendarmerie d'Espagne doit déplorer 94 désertions, auxquelles il convient d'ajouter celles de quarante-trois gendarmes cantabres. SHD-DAT, Xf 173 à 183bis.

29.Pour réaliser ces tableaux, nous nous sommes reportés aux états de situations de troupes françaises conservés au SHD-DAT dans les cartons suivants : pour l'Aragon, C8 386 ; pour la Navarre, C8 387 ; pour le gouvernement de Biscaye, C8 388 ; et pour Santander, C8 389.

30.Le décret de création de la 1ère légion de gendarmerie d'Espagne prévoit non seulement le prélèvement de quarante cavaliers par escadron mais également une augmentation du nombre de fantassins, le faisant passer de cent vingt à cent quatrevingts hommes. Les effectifs globaux d'un escadron passent donc à 224 hommes au lieu des deux cent sept, initialement prévus par le décret du 24 novembre 1809. Décret du 13 novembre 1810 portant création de la légion de gendarmerie à cheval de l'armée d'Espagne. SHD-DAT, Xf 166.

31.Par manque de chiffres précis sur le nombre de gendarmes à cheval présents dans les escadrons, nous avons privilégié le nombre de chevaux, seule valeur exacte permettant de quantifier la proportion de membres de l'arme effectivement montés. 32." Si on laisse la province [de Biscaye] avec les seuls gendarmes, elle courrera [sic.] le danger d'être envahie par les bandits qui l'entourent ». Rapport du général Avril du 8 mars 1810. SHD-DAT, C8 43.

33.« Abandonnée à elle-même, la Gendarmerie d'Aragon n'est pas assez forte pour résister avec avantage aux bandes de la Navarre réunies à celles de l'Aragon ». C'est dans cette configuration que la garnison de Huesca est tombée aux mains des insurgés en janvier 1812. Lettre du 21 janvier 1812 du général Buquet au maréchal Berthier. SHD-DAT, C8 89.

34.À son départ pour la province de Valence, Suchet ne laisse en Aragon que des unités en nombre restreint et les gendarmes considérant que « quelques escadrons de gendarmerie suffiraient pour le moment [sur la rive gauche de l'Ebre] ». Lettre du 14 octobre 1810 du général Suchet au général Pâris. CHAN, 384 AP 20. Pourtant, il se plaint peu de temps après auprès de Berthier « que jusqu'à ce jour le service de la Gendarmerie a été insignifiant en Aragon ». Lettre du 14 janvier 1811 du général Suchet au maréchal Berthier. SHD-DAT, C8 63. Si Suchet n'a pas surestimé la gendarmerie, n'at-il pas un tant soit peu sous-estimé la force des insurgés aragonais et navarrais? 35.Pour plus de détails sur les missions combattantes de la gendarmerie, voir Gildas LEPETIT, « La Gendarmerie impériale au combat, l'exemple de l'Espagne (1809-1814) », Revue historique des Armées, $\mathrm{n}^{\circ} 241$, 2005, p. 80-90. Pour les missions d'ordre économique, 
voir Gildas LEPETIT, « Gendarmerie et guerre économique dans les provinces septentrionales de l'Espagne (novembre 1809-juin 1813) ", dans Aspects économiques de la défense à travers les grands conflits mondiaux, actes du XXXe Congrès international d'histoire militaire, Rabat, août 2004, 2005, p. 417-424.

36. Ces compagnies sont créées le 1er mars 1811. Arrêté de Suchet du 31 mars 1811. AN, 384 AP 40. Bien que dénommés ainsi, les gendarmes aragonais ne font pas partie des escadrons de gendarmerie d'Espagne. Ils ont une gestion propre, bien qu'ils aient des avantages et des soldes proches de ceux des gendarmes français. Voir lettre du 2 décembre 1811 du commissaire des guerres Perrin à Musnier. AN, 384 AP 139. 37.Cette mesure concerne les 1er, $2 \mathrm{e}, 3 \mathrm{e}$ et $4 \mathrm{e}$ escadrons stationnés dans le $4 \mathrm{e}$ gouvernement militaire correspondant aux Provinces basques espagnoles. Pour plus de précisions sur les gendarmes cantabres et leur recrutement, voir Gildas LEPETIT, $L a$ Gendarmerie impériale dans le Vascongadas...op. cit., p. 146-159.

38.Rapport du 14 juin 1813 du ministre de la Guerre à l'empereur. AN, AF IV 1634. 39.Ils sont au nombre de quarante-trois. Il convient de préciser que le recrutement initial des gendarmes cantabres porte sur une centaine de personnes. Bien que des enrôlements complémentaires aient eu lieu sporadiquement, la proportion demeure considérable puisqu'elle atteint presque un Cantabre sur deux. SHD-DAT, Xf 173 à 183 bis.

40.Les états de situation ne mentionnent pas les causes des décès dans les cas de mort à l'hôpital.

41.Situation au 1er juin 1813 des escadrons de gendarmerie placés dans l'arrondissement de l'armée du nord de l'Espagne à l'époque. SHD-DAT, C8 109. 42.État nominatif du 8 janvier 1814 des officiers, sous-officiers et gendarmes cantabres espagnols existant dans les quatre légions de gendarmerie d'Espagne, dirigés sur Libourne en vertu des ordres de Son Excellence le maréchal duc de Dalmatie commandant l'armée de Sa Majesté l'Empereur en Espagne et aux Pyrénées. SHD-DAT, Xf 163.

43.Barroutia est jugé " dangereux et remuant ». Il est retenu au dépôt de l'île d'oléron qui est considérée à l'époque comme un « dépôt de punition ». État du 10 décembre 1816 des réfugiés espagnols militaires qui font partie du dépôt de l'Ile d'oléron. SHDDAT, Yj 125. De même, il a démontré à plusieurs reprises « son opposition au gouvernement légitime ». Note du 9 mai 1817 du colonel chef de l'état major de la division militaire de Tours. SHD-DAT, Yj 125.

44.Sur la question du renseignement, voir Gildas LEPETIT, « La Gendarmerie impériale au combat... » op. cit. p. 85-86.

45.Lettre du 20 avril 1810 du général Thouvenot au chef d'escadron Burette, commandant le 3e escadron. SHD-DAT, C8 185.

46.Lettre du 18 septembre 1811 du général Reille au maréchal Berthier. SHD-DAT, C8 81.

47.Le 4 novembre 1793 Turreau de Garambouville Louis Marie (1756-1816), commandant en chef de l'armée de l'Ouest, annonce par voie d'affiche : «Tous les brigands qui seront trouvés les armes à la main ou convaincus de les avoir prises pour se révolter contre leur patrie seront passés au fil de la baïonnette ». Cité par Jean TRANIÉ et Juan Carlos CARMIGNIANI, Les guerres de l'Ouest 1793-1815, Paris, éd. Lavauzelle, 1983, p. 195.

48.Lettre du 20 janvier 1812 du général Thouvenot au maréchal Berthier. SHD-DAT, C8 89. 
49.Rapport du 11 au 15 juillet 1812 du général Buquet au ministre de la Police générale. AN, F7 3049.

50.Lettre du 4 juin 1810 du général Thouvenot au général Buquet. SHD-DAT, C8 185.

51.Lettre du 14 janvier 1811 du général Suchet au maréchal Berthier. SHD-DAT, C8 63.

52.Rapport du 5 février 1810 du général Thouvenot. SHD-DAT, C8 139.

53.Rapport du 17 mars 1810 du général Buquet. SHD-DAT, C8 139.

54.Compte rendu de l'interrogatoire de Mina à Bayonne le 9 avril 1810. SHD-DAT, C8 45.

55.Lettre du 14 février 1811 du duc d'Istrie au maréchal Berthier. SHD-DAT, C8 65.

Lettre du18 juin 1811 de Buquet au ministre de la Guerre. SHD-DAT, C8 74.

56.Rapport du 2 mai 1813 du général Buquet à l'empereur. SHD-DAT, C8 141.

57.État des troupes insurgées qui sont dans les provinces du nord, mai 1813. SHD-DAT, C8 108.

58.Deux en janvier, une en février, une en mars et une en avril. Pour plus de détails, voir Gildas LEPETIT, La Gendarmerie impériale dans le Vascongadas...op. cit., p. 131-134.

59.Emmanuel Martin évalue à vingt-sept officiers et huit cent quatre sous-officiers le nombre de gendarmes tués en Espagne. Il comprend dans ce chiffre les hommes morts au sein des forces publiques et des légions de Burgos et de Catalogne. Les états de situations et les carnets d'état civil en confirment 784 pour les seuls escadrons. Mais certaines données ne nous sont pas parvenues. Ainsi celles du 14e escadron n'ont pu être retrouvées. De même, les $16 \mathrm{e}$ et $17 \mathrm{e}$ escadrons présentent des états incomplets. Si ceux du 16e ont pu être complétés par recoupement avec d'autres sources, il n'en est pas de même pour le 17e qui ne présente les morts qu'à compter du 28 janvier 1813. SHD-DAT, Xf 173 à 183bis. SHD-DAT, Xz 19.

60.800 morts sur 6500 hommes, soit $12,3 \%$.

61.Selon lui, la guérilla aurait tué, en moyenne, dix-huit mille soldats impériaux par an. Cité par Vittorio SCOTTI DougLAS, «La guérilla espagnole dans la guerre contre l'armée Napoléonienne ", dans Annales historiques de la Révolution française, 2004, n 2, p. 97. 62.Rory MuIR, Britain and the Defeat of Napoleon 1807-1815, New Haven and London, Yale University Press, 1996, p. 421.

63.Lettre du 26 juin 1812, Correspondance de Napoléon Ier..., op. cit., t. XXIII

64.Ainsi, le général Reille se plaint du coût de la gendarmerie, précisant que « huit cents gendarmes coûtent autant de solde que six bataillons d'infanterie » et rendent beaucoup moins de service, sous-entendant qu'il préférerait qu'on lui expédie des fantassins. Lettre du 4 décembre 1810 du général Reille au maréchal Berthier. SHD-DAT, C8 268.

\section{RÉSUMÉS}

Le 24 novembre 1809, Napoléon décrète la création de vingt escadrons de gendarmerie impériale destinés à servir dans les provinces septentrionales de l'Espagne. Ce corps, fort de plus de quatre mille hommes, s'établit sur plusieurs régions : la province de Santander, les provinces basques, la Navarre, l'Aragon, la Castille, puis la Catalogne. Les gendarmes ne quittent la péninsule qu'après la bataille de Vitoria (21 juin 1813). 
Au cours de cette période, la gendarmerie d'Espagne a œuvré quotidiennement au maintien des communications et au rétablissement d'un ordre mis à mal par les agissements des guérilleros. Pourtant, elle demeure pendant très longtemps dans l'ombre, n'étant qu'à de très rares exceptions mentionnée dans les études consacrées à la campagne d'Espagne.

Dès lors, il convient de s'intéresser aux silences de l'histoire en explicitant la place réelle de la gendarmerie impériale dans le dispositif français présent dans les provinces du nord de l'Espagne, tant en matière d'effectifs, d'activités que d'efficacité.

The most efficient way of maintaining tranquility? The role of the Imperial Gendarmerie in the French system in northern Spain (1810-1814). On November 24, 1809, Napoleon issued a decree creating twenty units of imperial gendarmerie to serve in the northern provinces of Spain. This corps, composed of more than four thousand men, was established in several regions: the province of Santander, the Basque Provinces, Navarre, Aragon, Castille, and Catalonia. The gendarmes left the peninsula only after the Battle of Vitoria (June 21 1813). During this period, the Spanish gendarmerie worked daily for the maintenance of communications and the reestablishment of order disturbed by the activities of the guerilleros. Yet the history of this gendarmerie has been long overlooked, and has rarely been mentioned in studies of the Spanish campaign. This article examines the role of the imperial gendarmerie in the French presence in the northern provinces of Spain, its size, its activity, and its efficiency.

\section{INDEX}

Mots-clés : Guerre d'Espagne, gendarmerie, mémoire, missions combattantes, maintien de l'ordre, justice prévôtale

\section{AUTEUR}

\section{GILDAS LEPETIT}

Château de Vincennes - BP 16600468 Armées, Délégation au Patrimoine culturel de la Gendarmerie nationale, shgm-gendarmerie@wanadoo.fr 